\title{
MEDICINE STORIES
}


This page intentionally left blank 
- Revised and Expanded Edition •

\section{MEDICINE STORIES \\ essays for radicals}

Aurora Levins Morales

Duke University Press | Durham and London | 2019 
Medicine Stories was originally published by South End Press, Cambridge, MA, 1998 Republished by Duke University Press, 2019

All rights reserved

Printed in the United States of America on acid-free paper $\infty$

Designed by Julienne Alexander

Typeset in Whitman and Meta Pro by Westchester Publishing Services

Library of Congress Cataloging-in-Publication Data

Names: Levins Morales, Aurora, [date] author.

Title: Medicine stories : essays for radicals / Aurora Levins Morales.

Description: Revised and expanded edition. | Durham : Duke University Press, 2019. |

Medicine Stories originally published by South End Press, Cambridge, MA, 1998;

republished by Duke University Press, 2019. | Includes bibliographical references and index.

Identifiers: LCCN 2018032571 (print)

LCCN 2018041258 (ebook)

ISBN 9781478003373 (ebook)

ISBN 9781478001904 (hardcover : alk. paper)

ISBN 9781478003090 (pbk. : alk. paper)

Subjects: LCSH: Levins Morales, Aurora, 1954- | Authors, American-

2oth century-Biography. | Women historians-United States-Biography. |

Historians-United States-Biography. | Feminists-United States-Biography. |

Jews-Puerto Rico-Biography. | Puerto Rico-Ethnic relations.

Classification: LCC PS3562.E915 (ebook) | LCC PS3562.E915 Z468 2019 (print) |

DDC $814 / .54$ [в]-dc23

LC record available at https://lccn.loc.gov/2018032571

"Libation," "Ecology Is Everything," "Bigger Is Better," "My Feminism," "Identity and Solidarity," "The Power of Story," "The Truths Our Bodies Tell," "False Memories," "Raícism," "The Politics of Childhood," "Forked Tongues," "Ban Me!," "Taíno Citizenship," "Speaking of Antisemitism," "BDS and Me," "Torturers," "Histerimonia," "Building Radical Soil," "Circle Unbroken," "Tai" () 2019 Aurora Levins Morales

Cover art by Ricardo Levins Morales 